\begin{tabular}{|c|l|}
\hline Title & Core swelling in spherical nuclei : A n indication of the saturation of nuclear density \\
\hline Author(s) & Horiuchi, W.; Inakura, T. \\
\hline Citation & $\begin{array}{l}\text { Physical Review C, 101(6), 061301 } \\
\text { https://doi.org/_0.1103/PhysRevC.101.061301 }\end{array}$ \\
\hline Issue Date & 2020-06-11 \\
\hline Doc URL & http://hdl.handle.net/2115//8944 \\
\hline Rights & @2020 A merican Physical Society \\
\hline Type & article \\
\hline File Information & PhysRevC.101-6_061301.pdf \\
\hline
\end{tabular}

Instructions for use 


\title{
Core swelling in spherical nuclei: An indication of the saturation of nuclear density
}

\author{
W. Horiuchi $\odot^{1, *}$ and T. Inakura ${ }^{2}$ \\ ${ }^{1}$ Department of Physics, Hokkaido University, Sapporo 060-0810, Japan \\ ${ }^{2}$ Laboratory for Advanced Nuclear Energy, Institute of Innovative Research, Tokyo Institute of Technology, Tokyo 152-8550, Japan
}

(Received 6 April 2020; accepted 28 May 2020; published 11 June 2020)

\begin{abstract}
Background: Nuclear radius is one of the most important and basic properties of atomic nuclei and its evolution is closely related to the saturation of the nuclear density in the internal region but the systematics of the nuclear radii for the neutron-rich unstable nuclei is not well known.

Purpose: Motivated by the recent interaction cross section measurement which indicates the ${ }^{48} \mathrm{Ca}$ core swelling in the neutron-rich $\mathrm{Ca}$ isotopes, we explore the mechanism of the enhancement of the neutron and proton radii for spherical nuclei.

Methods: Microscopic Hartree-Fock calculations with three sets of Skyrme-type effective interactions are performed for the neutron-rich $\mathrm{Ca}, \mathrm{Ni}$, and $\mathrm{Sn}$ isotopes. The total reaction cross sections for the $\mathrm{Ca}$ isotopes are evaluated with the Glauber model to compare them with the recent cross section data.

Results: We obtain good agreement with the measured cross sections and charge radii. The neutron and proton radii of the various "core" configurations are extracted from the full Hartree-Fock calculation and discuss the core swelling mechanism.

Conclusions: The core swelling phenomena occur depending on the properties of the occupying valence singleneutron states to minimize the energy loss that comes from the saturation of the densities in the internal region, which appears to be prominent in light nuclei such as $\mathrm{Ca}$ isotopes.
\end{abstract}

DOI: 10.1103/PhysRevC.101.061301

Introduction. Systematic studies of the nuclear density and radius give us an insight not only into the nuclear structure such as halo [1], neutron skin [2], and nuclear deformation [3], but also into the nuclear matter properties [4-9]. The electron scattering measurements, which precisely determine the charge distribution of nuclei have unveiled the nuclear saturation properties [10]. These studies have been extending to the short-lived neutron-rich unstable nuclei, in which the behavior of the nuclear density is not well known. The size properties of the unstable nuclei have often been extracted from the total reaction or interaction cross section measurements since the discovery of the neutron halo structure in ${ }^{11} \mathrm{Li}$ [1], and have been reached at the heavier two-neutron halo nucleus ${ }^{22} \mathrm{C}$ [11,12], and neutron-rich $\mathrm{Ne}$ [3] and $\mathrm{Mg}$ [13] isotopes. Recently, a sudden increase of the interaction cross section beyond ${ }^{48} \mathrm{Ca}$ was reported indicating the swelling of ${ }^{48} \mathrm{Ca}$ core in the $\mathrm{Ca}$ isotopic chain [14]. It has been recognized that the sudden enhancement of the matter radius is due to nuclear deformation $[3,13,15-19]$ or weakly bound neutrons like the halo structure $[1,11,13]$. However, these $\mathrm{Ca}$ isotopes are known to be spherical [20-22], and they are well bound in ${ }^{42-51} \mathrm{Ca}, S_{n} \approx 5-6 \mathrm{MeV}$ [23]. This may call a new mechanism for the enhancement of the nuclear size.

In this Rapid Communication, we aim to uncover the core swelling mechanism in light-medium to medium spherical nuclei and discuss how the "core" nucleus is developed as a

*whoriuchi@nucl.sci.hokudai.ac.jp function of the neutron number within a microscopic meanfield theory, that is, Skyrme-Hartree-Fock (HF) theory. We investigate even-even proton closed-shell isotopes, i.e., Ca, $\mathrm{Ni}$, and $\mathrm{Sn}$ isotopes, which exhibit almost spherical shape, and discuss the evolution of the size of the "core" nuclei. The core swelling phenomena may appear in the isotopic chain across the major shell or subshell. For example, the abrupt interaction cross section enhancement from ${ }^{22} \mathrm{O}(N=$ 14 subshell) to ${ }^{23} \mathrm{O}$ was found in Refs. [24,25]. The core swelling in $\mathrm{O}$ isotopes was theoretically discussed using ${ }^{16} \mathrm{O}$ plus few-neutron models in Ref. [26]. This study also helps formulate "core" plus few-nucleon models towards describing the light-medium mass dripline nuclei, e.g., ${ }^{22} \mathrm{C}[27,28]$, and ${ }^{29,31} \mathrm{~F}[29,30]$.

Theoretical models. Here, we perform the Skyrme-HF calculation and give a brief description of its model setup. The numerical code used in the present calculation is a revised version of the code developed in Refs. [18,31]. The groundstate wave function is obtained by minimizing the following energy density functional [32], $E[\rho]=E_{N}+E_{C}-E_{\text {c.m. }}$., using the imaginary-time method [33]. The nuclear energy $E_{N}$ is given by a functional of the nucleon density $\rho_{q}(\boldsymbol{r})$, the kinetic density $\tau_{q}(\boldsymbol{r})$, and the spin-orbit-current density $\boldsymbol{J}_{q}(\boldsymbol{r})(q=$ $n, p)$. The Coulomb energy $E_{C}$ among protons is a sum of direct and exchange parts. The exchange part is approximated by means of the Slater approximation, $\propto \int \mathrm{d} \boldsymbol{r} \rho_{q}(\boldsymbol{r})^{4 / 3} . E_{\mathrm{c} . \mathrm{m}}$. is the center of mass energy. The single-particle wave function $\phi_{i}(\boldsymbol{r})$ is represented in the three-dimensional grid points. All the single-particle wave functions and potentials except for the 
Coulomb potential are assumed to vanish outside the sphere of radius $20 \mathrm{fm}$. For the calculation of the Coulomb potential, we follow the prescription given in Ref. [34]. In order to see the model dependence, we employ three kinds of Skyrme parameter sets, SkM* [35], SLy4 [36], and SkI3 [37].

In this study, for the sake of simplicity, we only consider spherical configurations and neglect the pairing correlation. The pairing correlation makes it difficult to define the "core" configuration because this induces the fractional occupation probability. It should be noted that for nonclosed subshell nuclei, the HF calculation produces slightly deformed ground states, whereas the HF + BCS and Hartree-Fock-Bogoliubov calculations give spherical ground states due to the pairing correlations $[20,21,38,39]$. To preserve the spherical symmetry, we employ the filling approximation [40]. Namely, when the Fermi level with angular momentum $j$ is occupied partially by $m$ particles $(m<2 j+1)$, we consider that $2 j+1$ particles with the uniform occupation probability $m /(2 j+1)$ occupy the Fermi level. We note that the effect of the pairing interaction on the nuclear radii is small for a spherical ground state as was shown in Ref. [38].

Results and discussions. First we compute the total reaction cross sections of $\mathrm{Ca}$ isotopes following the prescriptions given in Ref. [18]. The nucleon-target formalism in the Glauber theory [41] (NTG [42]) is employed for the evaluation of the total reaction cross sections. Inputs to this reaction theory are the neutron and proton densities of projectile and target nuclei as well as the profile function which describes the nucleon-nucleon scattering at forward angles. The neutron and proton densities of the projectile nuclei are obtained from the $\mathrm{HF}$ calculations. For the target nucleus, ${ }^{12} \mathrm{C}$, we adopt the harmonic-oscillator density [43] whose width parameter is fixed so as to reproduce the root-mean-square (rms) pointproton radius of ${ }^{12} \mathrm{C}, 2.33 \mathrm{fm}$, extracted from the charge radius data [44]. Standard parameter sets of the profile function are tabulated in Ref. [45]. Once they are set, this model has no adjustable parameter and gives a nice description in highenergy nucleus-nucleus collisions as demonstrated in many examples [18,27,43,46-48].

Figure 1 plots the calculated total reaction cross sections of ${ }^{40-70} \mathrm{Ca}$ isotopes on a ${ }^{12} \mathrm{C}$ target at the incident energy of $280 \mathrm{MeV} /$ nucleon. Recent experimental interaction cross section data of ${ }^{42-51} \mathrm{Ca}$ [14] are also plotted for comparison. The calculated total reaction cross sections presented here fairly well reproduce the recent experimental data [14], which confirm the validity of the calculated matter densities. The interaction dependence of those adopted Skyrme parameter sets on the cross sections are small in the regions where the experimental data are available. The SkI3 interaction predicts somewhat larger cross sections for $N \gtrsim 30$ because SkI3 has larger slope parameter of the equation of the state, the so-called $L$, compared to others [49], leading to more rapid growth of the neutron skin thickness with respect to an increase of the neutron number [39]. The results of the neutron number $N>50$ are not shown although the calculation still produces several bound ground states with $N>50$ depending on the interaction employed. Note that the heaviest $\mathrm{Ca}$ isotope found so far is ${ }^{60} \mathrm{Ca}[50]$, and the recent theoretical works

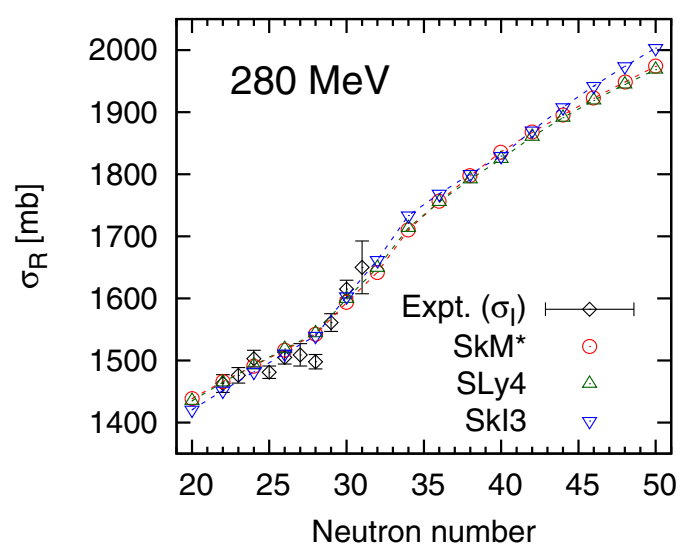

FIG. 1. Total reaction cross sections $\left(\sigma_{R}\right)$ of Ca isotopes on a ${ }^{12} \mathrm{C}$ target incident at $280 \mathrm{MeV} /$ nucleon. The $\mathrm{SkM}^{*}$, SLy4, and SkI3 interactions are employed for the HF calculations. Experimental interaction cross section $\left(\sigma_{I}\right)$ data are taken from Ref. [14].

predict the neutron dripline is close to ${ }^{60} \mathrm{Ca}$ [51], and possibly at ${ }^{70} \mathrm{Ca}[52]$, and at ${ }^{64} \mathrm{Ca}[22]$.

Although there are small differences, overall, the calculated densities reasonably explain the behavior of the available experimental cross sections. We discuss the size properties of the $\mathrm{Ca}, \mathrm{Ni}$, and $\mathrm{Sn}$ isotopes using these ground-state wave functions obtained. We calculate ${ }^{40-70} \mathrm{Ca}(N=20-50)$, ${ }^{56-86} \mathrm{Ni}(N=28-58)$, and ${ }^{114-146} \mathrm{Sn}(N=64-96)$ to cover various single-neutron configurations across the major shells. Here, the "core" nucleus has to be defined in a reasonable way so that we can discuss its size evolution. For this purpose, we extract these single-particle orbits that corresponds to the closed shell or subshell neutron configurations from the full HF configurations. The rms radii for neutrons and protons are calculated for each "core" configuration and their evolution with respect to the neutron number is discussed.

Figure 2 draws the rms neutron and proton radii of the $\mathrm{Ca}, \mathrm{Ni}$, and $\mathrm{Sn}$ isotopes obtained from the HF calculations and those extracted from their core configurations. The results with the $\mathrm{SkM}^{*}$ interaction are presented. The rms point-proton radii extracted from the charge radii [44] are also plotted for comparison and are found to be in good agreement with the theoretical calculations. A kink behavior found at $N=28$ of the $\mathrm{Ca}$ isotopes is qualitatively reproduced. Almost the same results are obtained with the SLy4 and SkI3 interactions. For more qualitative understanding of the kink behavior of the nuclear radii, we remark that the importance of the spinorbit interaction was discussed in Refs. [53-55]. The kink behaviors of the proton radii at $N=28$ in the Ca isotopes and at $N=126$ in the $\mathrm{Pb}$ isotopes were well reproduced by introducing density-dependent spin-orbit interaction. The spin-orbit interaction could modify the single-particle orbits and plays a role in the core swelling phenomena.

First, let us discuss the size evolution of the core nuclei in the $\mathrm{Ca}$ isotopes drawn in Fig. 2(a). As the neutron number increases, the total neutron radius gradually increases showing some kink behavior at the neutron numbers where the outermost single-neutron orbit is fully occupied. In general, since 

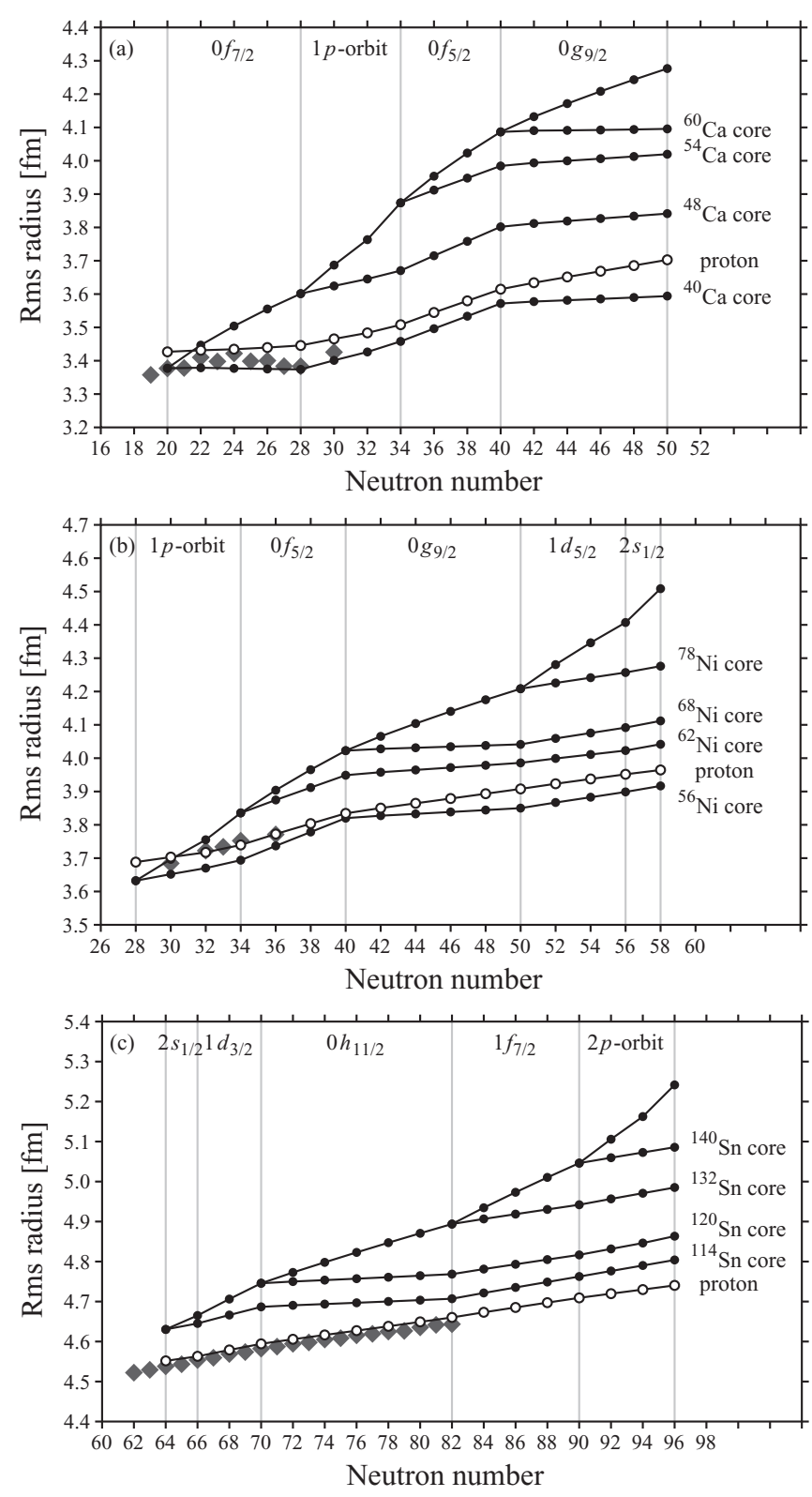

FIG. 2. Nuclear rms radii of (a) $\mathrm{Ca}$, (b) $\mathrm{Ni}$, and (c) $\mathrm{Sn}$ isotopes for neutron (closed circles) and proton (open circles). The rms neutron radii of various "core" nuclei are also plotted. The SkM* interaction is employed. See text for details. Filled diamonds in gray indicate experimental rms point-proton radii extracted from Ref. [44].

the centrifugal barrier is smaller, the lower angular momentum, the more diffused nuclear surface obtains [39], which leads to a larger rms radius. On the other hand, the neutron number dependence of the core nuclei behaves differently from that of the total neutron radii: From $N=20-28$ and $N=40-50$, the neutron radii of the ${ }^{40} \mathrm{Ca}$ and ${ }^{48} \mathrm{Ca}$ cores are almost constant, whereas from $N=28$ to 40 , they increase drastically as it almost follows the behavior of the proton radii. This fact is also consistent with the observation found in Ref. [14]. With large neutron excess at $N=40-50$, the proton radius extends for gaining the symmetry energy from the surrounding neutrons despite the fact that the neutron radius keeps its constant behavior. In fact, we see the linear enhancement of the proton radius following the enhancement of the neutron radius though the slope is not steeper than that of the neutron one.

In Fig. 2(b), similar behavior is also found in the $\mathrm{Ni}$ isotopes up to $N=50$ with further core swelling from $N=$ 50 to 58 . This isotopic chain extends to the $\mathrm{Sn}$ isotopes [Fig. 2(c)], showing the rapid increase of the neutron radius from $N=64-70$ and $N>82$, whereas the proton radii gradually increase without showing any kink behavior.

The enhancement of the neutron radius certainly reflects the properties of the outermost single-particle orbits. The constant behavior of the neutron core radius can only be found when the nodeless $j$-upper orbits such as $0 f_{7 / 2}, 0 g_{9 / 2}$, and $0 h_{11 / 2}$ orbits are occupied. When the enhancement of the neutron core radius occurs, the occupying orbits are always nodal wave functions such as $1 p, 1 d, 2 s, 1 f_{7 / 2}, 2 p$ orbits or $j$ lower orbits. Since these single-particle densities have strong overlap with the core density, the nuclear density in the internal region becomes high when nodal and $j$-lower orbits are added to the core nucleus, while the nodeless $j$-upper orbits only contribute to the densities at around the nuclear surface. If the internal density of the core is already saturated, the system looses the energy, and thus, to accommodate the additional neutrons, strong rearrangement of the mean field occurs to decrease the density in the internal region, which leads to the core swelling. We also made the same analysis for ${ }^{16-24} \mathrm{O}$. As expected from the above conjecture, the swelling of the ${ }^{16} \mathrm{O}$ core occurs from $N=14-16$ where the $1 s_{1 / 2}$ orbits contribute, which is consistent with the consequence given in Ref. [26].

To show the above finding more explicitly, in Fig. 3, we display the total matter (solid and long-dashed lines in black), core (dashed and dotted lines in red), and valence neutron densities (chain and double-dotted-spaced lines in blue) of the selected $\mathrm{Ca}, \mathrm{Ni}$, and $\mathrm{Sn}$ isotopes located in different shell or subshell regions. In the regions where the core swelling does not occur, which correspond to panels (a), (c), (e), and (h) of Fig. 3, the valence neutrons (chain and double-dotted-spaced lines) occupy the $j$-upper orbits and contribute mainly to the enhancement of the densities of the nuclear surface at around the nuclear half density. In case of ${ }^{50} \mathrm{Ca}$ and ${ }^{58} \mathrm{Ni}$ $(N=30)$, panels (b) and (d), respectively, we see that the central densities of the core (dotted line) are already saturated. The valence neutrons (double-dotted-spaced lines) occupy the nodal or $j$-lower orbits and contribute to the densities of all regions. By adding neutrons up to $N=40$, the central densities of the ${ }^{48} \mathrm{Ca}$ or ${ }^{56} \mathrm{Ni}$ core (dashed lines) have to be reduced to keep the saturation density $\approx 0.16-0.17 \mathrm{fm}^{-3}$ in the total matter densities (solid lines). Thus the core densities are enhanced in the surface region. In the panels (f), (g), and (i), the central densities of the core (dashed and dotted lines) becomes $\approx 0.15 \mathrm{fm}^{-3}$, which can accommodate further neutrons as they are indicated in the valence neutron densities (chain and double-dotted-spaced lines). In these isotopes, the matter radii are enhanced due to the contributions from both the core and valence neutron densities at the surface region. 


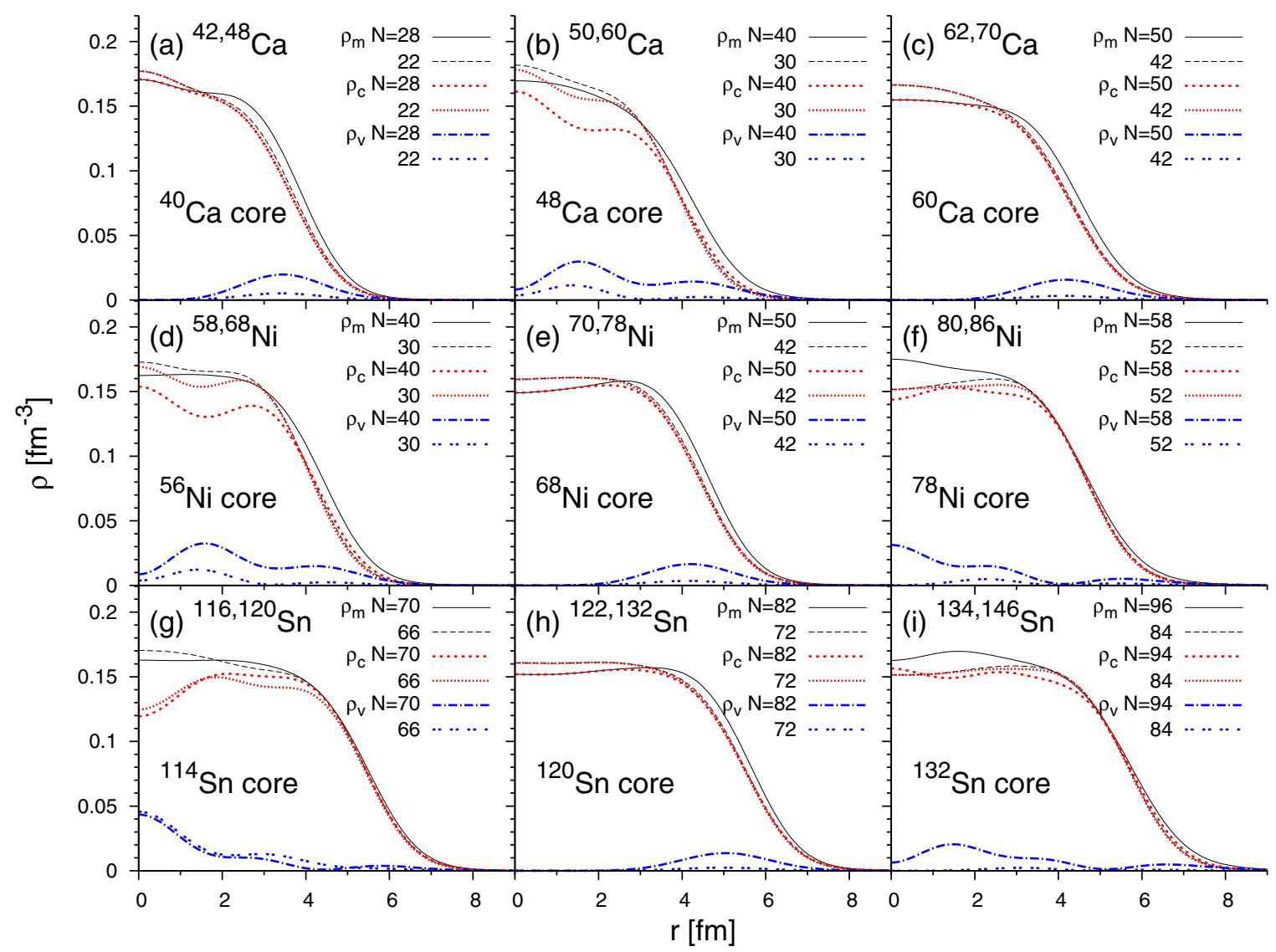

FIG. 3. Total matter $\left(\rho_{m}\right)$, core $\left(\rho_{c}\right)$, and valence neutron $\left(\rho_{v}\right)$ densities of ${ }^{42,48,50,60,62,70} \mathrm{Ca},{ }^{58,68,70,78,80,86} \mathrm{Ni}$, and ${ }^{116,120,122,132,134,146} \mathrm{Sn}$. The $\mathrm{SkM}^{*}$ interaction is employed. See text for details.

The internal nucleons are pulled by the excess neutrons to gain the symmetry energy as presented in the total matter densities (solid and long-dashed lines) in panels (e) and (h).

To strengthen the above conjecture, we perform another $\mathrm{HF}$ calculation for the valence neutrons by assuming an inert or frozen core in the system. Actually, we evaluate the energy difference between the HF calculation $E_{\mathrm{HF}}$ and the HF calculation with frozen core, $E_{\text {frozen }}$. In the frozen-core HF calculations, the wave function of the "core" nucleus is replaced by the ground-state wave function of the corresponding nucleus. For instance, the $E_{\text {frozen }}$ of ${ }^{52} \mathrm{Ca}$, which is assumed to have ${ }^{48} \mathrm{Ca}$ core and four valence neutrons, is calculated by optimization of the four valence neutron wave functions. The wave functions of the ${ }^{48} \mathrm{Ca}$ core is always represented by the ground state of ${ }^{48} \mathrm{Ca}$ obtained by the full $\mathrm{HF}$ calculation. No rearrangement among core and valence neutron configurations occurs in the frozen-core HF calculations. Therefore, the energy difference $E_{\text {frozen }}-E_{\mathrm{HF}}$ can be a measure of the mean-field rearrangement by adding the valence neutrons.

Figure 4 displays $E_{\text {frozen }}-E_{\mathrm{HF}}$ of the $\mathrm{Ca}, \mathrm{Ni}$, and $\mathrm{Sn}$ isotopes. No qualitative difference is found in the calculations with the SkM*, SLy4, and SkI3 interactions. As expected, the energy loss appears to be large when the nodal or $j$-lower orbits are occupied, especially, in $N=28-40$ for the Ca and $\mathrm{Ni}$ isotopes. On the other hand, the energy difference is small when the nodeless $j$-upper orbits, the $0 f_{7 / 2}, 0 g_{9 / 2}$, and $0 h_{11 / 2}$ orbits, are filling, in which no swelling the neutron core occurs as displayed in Fig. 2. This means that in those $N$ regions the core and valence neutrons densities are well separated in terms of the nuclear saturation.

We find a moderate energy loss in $N=50-58$ for the $\mathrm{Ni}$ isotopes and $N=64-70,82-96$ for the $\mathrm{Sn}$ isotopes despite the fact that a weak increase of the neutron core radius occurs as presented in panels (b) and (c) of Fig. 2. This is because the internal densities of those core nuclei, ${ }^{60} \mathrm{Ca}$ and ${ }^{114,132} \mathrm{Sn}$, are not well saturated as presented in panels (f), (g), and (i) of Fig. 3 (dashed lines) for $N=58, N=70$, and $N=84$, respectively. Since these internal densities are low enough to accommodate the additional neutrons that comes from the internal amplitudes of the nodal orbits, the rearrangement of the mean field is weaker, and thus the energy loss is suppressed.

Conclusions. The sudden increase of the nuclear radii is the indication of the saturation of the internal density balanced with the nuclear symmetry energy. It is found that the evolution of the neutron radius is strongly depend on the occupying valence neutrons. By adding the nodeless $j$-upper 

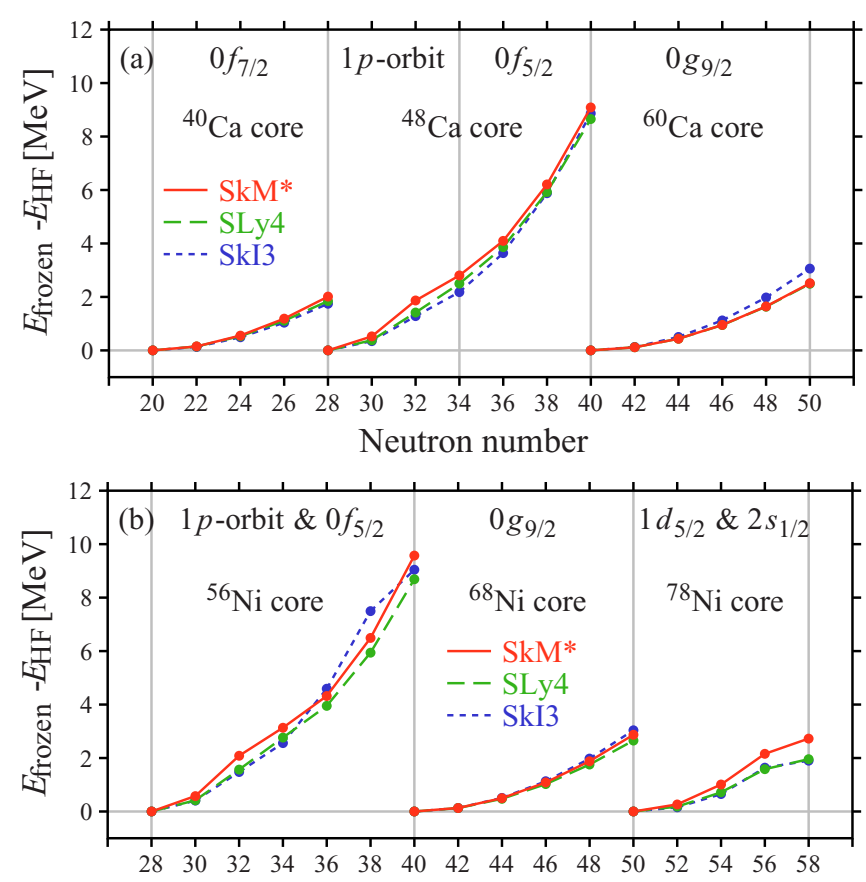

Neutron number

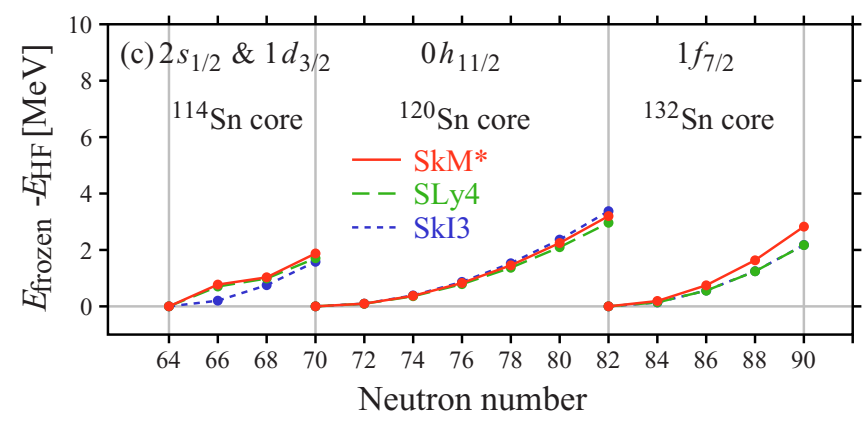

FIG. 4. Energy difference between the HF and the frozen-core $\mathrm{HF}$ calculations of (a) Ca, (b) $\mathrm{Ni}$, and (c) $\mathrm{Sn}$ isotopes with the $\mathrm{SkM}^{*}$, SLy4, and SkI3 interactions. See text for details. orbits, it shows the constant behavior of the neutron radii of the core nucleus because these orbits only contribute to the nuclear surface where the nuclear densities are unsaturated. A sudden increase of the neutron core radius occurs when the nodal or $j$-lower orbits are occupied. Strong rearrangement of the mean field happens to reduce the central density to accommodate these orbits that contribute to the densities of all regions. This behavior is significant in light-medium mass nuclei, such as $\mathrm{Ca}$ and $\mathrm{Ni}$ isotopes as the valence nucleons strongly influence the core configurations. For heavier nuclei, $\mathrm{Sn}$, the neutron and proton radii are determined in a more democratic way. Since more neutrons can be accommodated in the internal region, the moderate core swelling is expected for such nuclei with large neutron excess, where the internal densities are not well saturated.

This knowledge is also useful when one formulates a core plus few-nucleon model. When the valence nucleons occupy the nodal or $j$-lower orbits, some energy loss is expected if the core nucleus is inert or the core swelling is not introduced. However, this effect may be small in the case of the dripline nuclei because the core nucleus is expected to be already swelled and its internal density is unsaturated.

Systematic studies of the neutron and proton radii and densities are desired to reveal these nuclear size properties of finite nuclei. For this purpose, a systematic measurement of the total reaction cross sections on a proton target $[38,56]$ may be the most promising way to investigate the core swelling phenomena for the short-lived neutron-rich nuclei.

In this Rapid Communication, we only investigate the spherical nuclei for simplicity. A further study considering the nuclear deformation is interesting and will be reported elsewhere.

Acknowledgments. This work was in part supported by JSPS KAKENHI Grants No. 18K03635, No. 18H04569, and No. 19H05140. We acknowledge the collaborative research program 2020, information initiative center, Hokkaido University.
[1] I. Tanihata, H. Hamagaki, O. Hashimoto, Y. Shida, N. Yoshikawa, K. Sugimoto, O. Yamakawa, T. Kobayashi, and N. Takahashi, Phys. Rev. Lett. 55, 2676 (1985).

[2] T. Suzuki, H. Geissel, O. Bochkarev, L. Chulkov, M. Golovkov, D. Hirata, H. Irnich, Z. Janas, H. Keller, T. Kobayashi et al., Phys. Rev. Lett. 75, 3241 (1995).

[3] M. Takechi, T. Ohtsubo, T. Kuboki, M. Fukuda, D. Nishimura, T. Suzuki, T. Yamaguchi, A. Ozawa, T. Moriguchi, T. Sumikawa et al., Mod. Phys. Lett. A 25, 1878 (2010).

[4] L. W. Chen, C. M. Ko, B. A. Li, and J. Xu, Phys. Rev. C 82, 024321 (2010).

[5] P.-G. Reinhard and W. Nazarewicz, Phys. Rev. C 81, 051303(R) (2010).

[6] X. Roca-Maza, M. Centelles, X. Viñas, and M. Warda, Phys. Rev. Lett. 106, 252501 (2011).

[7] M. Kortelainen, J. Erler, W. Nazarewicz, N. Birge, Y. Gao, and E. Olsen, Phys. Rev. C 88, 031305(R) (2013).
[8] T. Inakura, T. Nakatsukasa, and K. Yabana, Phys. Rev. C 88, 051305(R) (2013).

[9] X. Roca-Maza, X. Viñas, M. Centelles, B. K. Agrawal, G. Coló, N. Paar, J. Piekarewicz, and D. Vretenar, Phys. Rev. C 92, 064304 (2015).

[10] H. de Vries, C. W. de Jager, and C. de Vries, At. Data Nucl. Data Tables 36, 495 (1987).

[11] K. Tanaka, T. Yamaguchi, T. Suzuki, T. Ohtsubo, M. Fukuda, D. Nishimura, M. Takechi, K. Ogata, A. Ozawa, T. Izumikawa et al., Phys. Rev. Lett. 104, 062701 (2010).

[12] Y. Togano, T. Nakamura, Y. Kondo, J. A. Tostevin, A. T. Saito, J. Gibelin, N. A. Orr, N. L. Achouri, T. Aumann, H. Baba et al., Phys. Lett. B 761, 412 (2016).

[13] M. Takechi, S. Suzuki, D. Nishimura, M. Fukuda, T. Ohtsubo, M. Nagashima, T. Suzuki, T. Yamaguchi, A. Ozawa, T. Moriguchi et al., Phys. Rev. C 90, 061305(R) (2014).

[14] M. Tanaka, M. Takechi, M. Fukuda, D. Nishimura, T. Suzuki, Y. Tanaka, T. Moriguchi, D. S. Ahn, A. Aimaganbetov, M. Amano et al., Phys. Rev. Lett. 124, 102501 (2020). 
[15] K. Minomo, T. Sumi, M. Kimura, K. Ogata, Y. R. Shimizu, and M. Yahiro, Phys. Rev. C 84, 034602 (2011).

[16] K. Minomo, T. Sumi, M. Kimura, K. Ogata, Y. R. Shimizu, and M. Yahiro, Phys. Rev. Lett. 108, 052503 (2012).

[17] T. Sumi, K. Minomo, S. Tagami, M. Kimura, T. Matsumoto, K. Ogata, Y. R. Shimizu, and M. Yahiro, Phys. Rev. C 85, 064613 (2012).

[18] W. Horiuchi, T. Inakura, T. Nakatsukasa, and Y. Suzuki, Phys. Rev. C 86, 024614 (2012).

[19] S. Watanabe, K. Minomo, M. Shimada, S. Tagami, M. Kimura, M. Takechi, M. Fukuda, D. Nishimura, T. Suzuki, T. Matsumoto et al., Phys. Rev. C 89, 044610 (2014).

[20] M. V. Stoitsov, J. Dobaczewski, W. Nazarewicz, S. Pittel, and D. J. Dean, Phys. Rev. C 68, 054312 (2003).

[21] J.-P. Delaroche, M. Girod, J. Libert, H. Goutte, S. Hilaire, S. Péru, N. Pillet, and G. F. Bertsch, Phys. Rev. C 81, 014303 (2010).

[22] S. Tagami, M. Tanaka, M. Takechi, M. Fukuda, and M. Yahiro, Phys. Rev. C 101, 014620 (2020).

[23] M. Wang, G. Audi, F. G. Kondev, W. J. Huang, S. Naimi, and X. Xu, Chin. Phys. C 41, 030003 (2017).

[24] A. Ozawa, T. Kobayashi, T. Suzuki, K. Yoshida, and I. Tanihata, Phys. Rev. Lett. 84, 5493 (2000).

[25] R. Kanungo, A. Prochazka, M. Uchida, W. Horiuchi, G. Hagen, T. Papenbrock, C. Nociforo, T. Aumann, D. Boutin, D. CortinaGil et al., Phys. Rev. C 84, 061304(R) (2011).

[26] H. Masui, K. Katō, and K. Ikeda, Nucl. Phys. A 895, 1 (2012).

[27] W. Horiuchi and Y. Suzuki, Phys. Rev. C 74, 034311 (2006).

[28] J. Singh, W. Horiuchi, L. Fortunato, and A. Vitturi, Few-Body Syst. 60, 50 (2019).

[29] J. Singh, J. Casal, W. Horiuchi, L. Fortunato, and A. Vitturi, Phys. Rev. C 101, 024310 (2020).

[30] H. Masui, W. Horiuchi, and M. Kimura, Phys. Rev. C 101, 041303(R) (2020).

[31] T. Inakura, H. Imagawa, Y. Hashimoto, S. Mizutori, M. Yamagami, and K. Matsuyanagi, Nucl. Phys. A 768, 61 (2006).

[32] D. Vautherin and D. M. Brink, Phys. Rev. C 5, 626 (1972).

[33] K. T. R. Davies, H. Flocard, S. Krieger, and M. S. Weiss, Nucl. Phys. A 342, 111 (1980).

[34] H. Flocard, S. E. Koonin, and M. S. Weiss, Phys. Rev. C 17, 1682 (1978).

[35] J. Bartel, P. Quentin, M. Brack, C. Guet, and H. Håkansson, Nucl. Phys. A 386, 79 (1982).
[36] E. Chanbanat, P. Bonche, P. Haensel, J. Mayer, and R. Schaeffer, Nucl. Phys. A 627, 710 (1997).

[37] P.-G. Reinhard and H. Flocard, Nucl. Phys. A 584, 467 (1995)

[38] W. Horiuchi, S. Hatakeyama, S. Ebata, and Y. Suzuki, Phys. Rev. C 93, 044611 (2016).

[39] W. Horiuchi, S. Ebata, and K. Iida, Phys. Rev. C 96, 035804 (2017).

[40] M. Beiner, H. Flocard, N. V. Giai, and Ph. Quentin, Nucl. Phys. A 238, 29 (1975).

[41] R. J. Glauber, in Lectures in Theoretical Physics, edited by W. E. Brittin and L. G. Dunham (Interscience, New York, 1959), Vol. 1, p. 315.

[42] B. Abu-Ibrahim and Y. Suzuki, Phys. Rev. C 61, 051601(R) (2000).

[43] B. Abu-Ibrahim, S. Iwasaki, W. Horiuchi, A. Kohama, and Y. Suzuki, J. Phys. Soc. Jpn. 78, 044201 (2009).

[44] I. Angeli and K. P. Marinova, At. Data Nucl. Data Tables 99, 69 (2013).

[45] B. Abu-Ibrahim, W. Horiuchi, A. Kohama, and Y. Suzuki, Phys. Rev. C 77, 034607 (2008); 80, 029903(E) (2009); 81, 019901(E) (2010).

[46] W. Horiuchi, Y. Suzuki, P. Capel, and D. Baye, Phys. Rev. C 81, 024606 (2010).

[47] W. Horiuchi, Y. Suzuki, B. Abu-Ibrahim, and A. Kohama, Phys. Rev. C 75, 044607 (2007); 76, 039903(E) (2007).

[48] W. Horiuchi, T. Inakura, T. Nakatsukasa, and Y. Suzuki, JPS Conf. Proc. 6, 030079 (2015).

[49] M. Dutra, O. Lourenço, J. S. Sá Martins, A. Delfino, J. R. Stone, and P. D. Stevenson, Phys. Rev. C 85, 035201 (2012).

[50] O. B. Tarasov, D. S. Ahn, D. Bazin, N. Fukuda, A. Gade, M. Hausmann, N. Inabe, S. Ishikawa, N. Iwasa, K. Kawata et al., Phys. Rev. Lett. 121, 022501 (2018).

[51] G. Hagen, M. Hjorth-Jensen, G. R. Jansen, R. Machleidt, and T. Papenbrock, Phys. Rev. Lett. 109, 032502 (2012).

[52] L. Neufcourt, Y. Cao, W. Nazarewicz, E. Olsen, and F. Viens, Phys. Rev. Lett. 122, 062502 (2019).

[53] H. Nakada and T. Inakura, Phys. Rev. C 91, 021302(R) (2015).

[54] H. Nakada, Phys. Rev. C 92, 044307 (2015).

[55] H. Nakada, Phys. Rev. C 100, 044310 (2019).

[56] W. Horiuchi, Y. Suzuki, and T. Inakura, Phys. Rev. C 89, 011601(R) (2014). 